\title{
Multifocal Motor Neuropathy
}

National Institute of Neurological Disorders and Stroke (NINDS)

\section{Source}

National Institute of Neurological Disorders and Stroke (NINDS). Multifocal Motor

Neuropathy Information Page.

Multifocal motor neuropathy is a progressive muscle disorder characterized by muscle weakness in the hands, with differences from one side of the body to the other in the specific muscles involved. It affects men much more than women. Symptoms also include muscle wasting, cramping, and involuntary contractions or twitching of the leg muscles. The disorder is sometimes mistaken for amyotrophic laterial sclerosis (ALS, or Lou Gehrig's disease) but unlike ALS, it is treatable. An early and accurate diagnosis allows patients to recover quickly. 